\title{
Fast and famous: looking for the fastest speed at which a face can be recognized
}

\author{
Gladys Barragan-Jason ${ }^{1}{ }^{*}$, Gabriel Besson ${ }^{1,2}$, Mathieu Ceccaldi ${ }^{2,3}$ and Emmanuel J. Barbeau ${ }^{1}$ \\ ' Centre de Recherche Cerveau et Cognition, Université de Toulouse, CNRS-UMR 5549, Toulouse, France \\ 2 Institut de Neurosciences des Systèmes, INSERM UMR1106, Aix-Marseille Université, Marseille, France \\ ${ }^{3}$ Assistance Publique Hôpitaux de Marseille, Service de Neurologie et Neuropsychologie, Centre Hospitalier Universitaire La Timone, Marseille, France
}

\section{Edited by:}

Linda Isaac, Palo Alto VA and Stanford University, USA

\section{Reviewed by:}

Anthony Little, University of Stirling, UK

Rick Thomas, University of Oklahoma, USA

\section{*Correspondence:}

Gladys Barragan-Jason, Centre de Recherche Cerveau et Cognition, CNRS CERCO UMR 5549, Centre Hospitalier Universitaire Purpan, Pavillon Baudot, BP 25202, 31052 Toulouse Cedex, France. e-mail: gladou86@gmail.com
Face recognition is supposed to be fast. However, the actual speed at which faces can be recognized remains unknown. To address this issue, we report two experiments run with speed constraints. In both experiments, famous faces had to be recognized among unknown ones using a large set of stimuli to prevent pre-activation of features which would speed up recognition. In the first experiment (31 participants), recognition of famous faces was investigated using a rapid go/no-go task. In the second experiment, 101 participants performed a highly time constrained recognition task using the Speed and Accuracy Boosting procedure. Results indicate that the fastest speed at which a face can be recognized is around $360-390 \mathrm{~ms}$. Such latencies are about $100 \mathrm{~ms}$ longer than the latencies recorded in similar tasks in which subjects have to detect faces among other stimuli. We discuss which model of activation of the visual ventral stream could account for such latencies. These latencies are not consistent with a purely feed-forward pass of activity throughout the visual ventral stream. An alternative is that face recognition relies on the core network underlying face processing identified in fMRI studies (OFA, FFA, and pSTS) and reentrant loops to refine face representation. However, the model of activation favored is that of an activation of the whole visual ventral stream up to anterior areas, such as the perirhinal cortex, combined with parallel and feed-back processes. Further studies are needed to assess which of these three models of activation can best account for face recognition.

Keywords: face recognition, familiarity, famous faces, go/no-go, SAB, speed constraints, memory

\section{INTRODUCTION}

The idea that face recognition is fast appears appealing (e.g., Bruce and Young, 1986; Jemel et al., 2010; Zheng et al., 2012). So what is the fastest speed at which a face can be recognized? Highly variable reaction times (RTs) have actually been reported in studies of face recognition, ranging from 400 to $900 \mathrm{~ms}$ (mean or median RTs, e.g., Kampf et al., 2002; Herzmann et al., 2004; Caharel et al., 2005; Anaki et al., 2007; Baird and Burton, 2008; Anaki and Bentin, 2009; Ramon et al., 2011; Barragan-Jason et al., 2012). Such variability can be accounted for by numerous factors, such as the number of stimuli (only one to hundreds), the use of repeated or trial-unique stimuli, and the nature of the stimuli (photographs or drawings). Moreover, the variety of protocols probably also accounts for some of this variability: yes/no (Caharel et al., 2007) but also priming (Lewis and Ellis, 2000) or categoryverification (Rosch, 1975; Anaki and Bentin, 2009) tasks have been used. Intriguingly, whereas it is common to use challenging tasks in the field of object recognition, with the aim to address the fastest speed at which objects can be recognized (e.g., go/no-go task in Thorpe et al., 1996), this appears less common in the field of face recognition.

"Face recognition" is an ambiguous term however, as it can refer to either "top-down" or "bottom-up face recognition." Top-down recognition corresponds to the situation of looking for someone in particular and involves the pre-activation of some diagnostic features about the person to be recognized (e.g., Lewis and Ellis, 2000; Tanaka, 2001). Such a paradigm can presumably be performed on the basis of a search for a few visual features and is expected to be fast. In contrast, bottom-up recognition corresponds to the situation of suddenly bumping into an acquaintance. This implies that subjects do not have any expectation about the face that will be presented, as in experiments where a large number of photographs with different identities are used. We can hypothesize that bottom-up recognition involves activation of representations in memory and thus would be rather slow. For example, in Lewis and Ellis (2000), different photographs of the same famous person (vs. another person) were presented and subjects had to press a different button for each identity. Authors reported that under such condition, face recognition was possible in $250 \mathrm{~ms}$ on average (after several repetitions) with an accuracy that could reach $100 \%$. Even if this was not explicit, this task is clearly based on top-down face recognition processes. In contrast, subjects performed in an experiment by Kampf et al. (2002) a manual yes/no task on 144 famous faces presented among 244 unknown ones. Mean RT was $431 \mathrm{~ms}$ with an accuracy that varied between 67 and 91\%. In this case, the task is based on bottom-up face recognition and RTs are much longer than in the previous study that relied on top-down face recognition. The fastest speed at which a face can be recognized thus clearly depends on the type of paradigm (top-down or bottom-up) used. 
The speed at which a face can be recognized also depends on the strategy used by subjects (Bentin and Deouell, 2000). In particular, recognizing a familiar face in a bottom-up manner can be based either on familiarity only or on identification, familiarity being faster than identification (Yovel and Paller, 2004) in accordance with hierarchical models of face recognition (Bruce and Young, 1986). Furthermore, it has been hypothesized that participants may have difficulties preventing identification, that is, it is not enough to find a face familiar, one has to retrieve some knowledge about the identity of the person to behave appropriately (Bruce and Young, 1986; Valentine, 2001). Hence, most RTs reported to date may be rather long. This suggests that the speed of the fastest answers in face recognition tasks cannot be properly assessed if time constraints are not used to try to prevent participants from accessing the identification level.

Overall, no clear picture has emerged about the real speed of face recognition, mainly because no speed constraints have been used to our knowledge. However, the fastest RTs reported to date are around $400 \mathrm{~ms}$. In Anaki et al. (2007), subjects had to categorize a large number of faces (180 famous and 180 unknown faces) using a yes/no paradigm. Mean RT was $411 \mathrm{~ms}$. In a recent study by Ramon et al. (2011), minimum (not mean) RTs were around $370 \mathrm{~ms}$ in a task in which subjects had to recognize personally known faces (28 classmates) among unknown matched faces. It can thus be hypothesized that face familiarity should be at least as fast as $400 \mathrm{~ms}$, and probably faster if speed constraints are used. Here, we report two experiments run with speed constraints and concentrate on bottom-up recognition. In the first experiment, participants had to recognize famous faces among unknown ones during a rapid go/no-go task. In the second experiment, we applied a new paradigm adding additional time constraints on responses.

\section{GENERAL METHODS \\ EXPERIMENTAL SETTING}

Participants sat in a dimly lit room, $90 \mathrm{~cm}$ from a CRT computer screen. Stimuli were presented using Eprime 2.0 software and subtended a visual angle of $\sim 7.2 \times 10.7$. The photographs were displayed on a black background. To answer, participants had to raise their fingers from an infrared response pad as quickly as possible (e.g., Rousselet et al., 2003).

\section{BEHAVIORAL PERFORMANCE ANALYSES}

Performance (accuracy) and bias were computed using $d^{\prime}$ and $C$ measures (Snodgrass and Corwin, 1988). Based on the Signal Detection Theory, these measures reflect respectively the discrimination performance between targets and distractors and the strategy used (positive if conservative, negative if liberal). As 0 and $100 \%$ cannot be $Z$-transformed, a correction was applied where appropriate following Snodgrass and Corwin (1988). As some participants failed to do the task $\left[\chi^{2}\right.$-test between hits and false alarms (FA), $p<0.05]$, their data were discarded from further analyses (see Table 1 for details). RTs $<200$ ms were considered as anticipation and were discarded from analyses (Rousselet et al., 2003; Barragan-Jason et al., 2012). To obtain an estimation of the minimal processing time required to recognize targets, the minimal behavioral reaction time (minRT) was computed by determining the latency at which correct go-responses (hits) started to significantly outnumber incorrect go-responses (FA; Rousselet et al., 2003; Bacon-Macé et al., 2007). For each condition, the analyses were performed either across trials (by pooling all trials from all participants for a given condition) and across participants (mean of all participants' individual mean performances). Across-trial analyses have been used in previous studies (Rousselet et al., 2003; Barragan-Jason et al., 2012; Besson et al.,

Table 1 | Results for each experiment.

\begin{tabular}{|c|c|c|c|}
\hline & & $\begin{array}{l}\text { Experiment 1: } \\
\text { speeded go/no-go }\end{array}$ & Experiment 2: SAB \\
\hline Succeeded on the task/underwent the task & $N(\%)$ & $31 / 31(100 \%)$ & $87 / 101(86.1 \%)$ \\
\hline \multirow[t]{4}{*}{ Accuracy $\left(d^{\prime}\right)$} & Median & 1.27 & 0.98 \\
\hline & First and third quartiles & {$[0.94 ; 1.51]$} & {$[0.59 ; 1.32]$} \\
\hline & Range & {$[0.38 ; 1.82]$} & {$[0.33 ; 2.22]$} \\
\hline & Across trials & 1.11 & 0.90 \\
\hline \multirow[t]{4}{*}{ Bias $(C)$} & Median & 0.55 & 0.07 \\
\hline & 1st and 3rd quartiles & {$[0.34 ; 0.85]$} & {$[-0.13 ; 0.39]$} \\
\hline & Range & {$[-0.13 ; 1.95]$} & {$[-1.00 ; 1.78]$} \\
\hline & Across trials & 0.54 & 0.09 \\
\hline Obtained a minRT & $N$ & $30 / 31(96.8 \%)$ & $57 / 87(65.5 \%)$ \\
\hline \multirow[t]{3}{*}{ Minimum RTs } & Median & 555 & 420 \\
\hline & First and Third quartiles & {$[510 ; 600]$} & {$[390 ; 480]$} \\
\hline & Range & {$[390 ; 780]$} & {$[360 ; 540]$} \\
\hline \multirow[t]{2}{*}{ Obtained a minRT at or before $420 \mathrm{~ms}$} & $N$ & $2 / 31(6.5 \%)$ & $32 / 87(36.8 \%)$ \\
\hline & Across trials & 450 & 350 \\
\hline
\end{tabular}

$M$, mean; N, number of participants; $R T$, reaction time; $S D$, standard deviation; minRT, minimal $R T$. 
2012) and are like building a "meta-participant," reflecting the performance over all the population. MinRTs across trials were computed using $10 \mathrm{~ms}$ time bins and determined as the middle of the first bin that was significant, $\chi^{2}$-test, $p<0.05$, followed by at least three significant consecutive bins. Across participants, in order to allow for the lower statistical power than with across-trial data since there were fewer trials, we used $30 \mathrm{~ms}$ time bins and a Fisher's exact test $(p<0.05)$, followed by at least two significant consecutive bins. Because of the non-normality of the data, Wilcoxon non-parametric tests (to compare bias of participants to 0 ) and non-parametric Spearman's rank correlation coefficient were used.

\section{EXPERIMENT 1: RAPID GO/NO-GO CATEGORIZATION TASK PARTICIPANTS}

Thirty-one participants [17 female, median age: 24, (range: [1937]), 27 right-handers] with normal or corrected-to-normal vision volunteered and gave their written informed consent to participate in the experiment.

\section{STIMULI}

Stimuli consisted of 540 photographs of unknown human faces and 270 photographs of famous faces (Brad Pitt, Bill Gates, etc.) chosen in a previous experiment as being recognized by people between 20 and 40 years old. All faces were presented in their natural context (i.e., they were not cropped and some background could be seen). Photographs of unknown faces were chosen to look like those of famous people in terms of quality (professional photographs), attractiveness (most of the photographs were of models) and emotion so that participants could not base their answers on these criteria. Each image was $320 \times 480$ pixels. Both series were comparable in luminance, contrast, number of pixels of the face in the image (manually cropped from their background). We also controlled other factors such as head orientation, emotion, and gaze (see supplementary Table 1 of Barragan-Jason et al., 2012). Examples of stimuli are provided in Figure A1A in Appendix.

\section{PROTOCOL}

Experiment 1 consisted of a go/no-go recognition task (Famous/non-Famous recognition task) divided into three blocks of 180 (90 targets randomly chosen from the famous face face stimulus set and 90 distractors randomly chosen from the unknown face stimulus set. Random selection of images was done individually for each participant). Participants were trained before each condition with a specific set of stimuli. Participants were instructed to raise their fingers from an infrared response pad (go response) as quickly as possible when a target (a famous face) was presented. At the beginning of each trial, a fixation cross appeared for a random interval (300-600 ms), followed by a photograph flashed for $100 \mathrm{~ms}$ and a black screen for $1000 \mathrm{~ms}$ (Figure 1A). Stimuli were randomly displayed in blocks and among participants.

\section{SPEED CONSTRAINTS}

Stimuli were flashed quickly $(100 \mathrm{~ms})$ and participants had to answer before $1000 \mathrm{~ms}$ post-stimulus. Participants performed training sessions with dedicated stimuli before each task and they could repeat training if they wanted. After each block, including the training session, mean RTs, and false-alarm rates were displayed so that participants could monitor their performance. Lastly, they received strong encouragement before and between blocks to answer as fast as possible. In particular, after each block, they were asked to "beat" their RT score.

\section{RESULTS}

Results across participants and across trials are presented in Table 1. Individual results are presented in Figures 2A,B. No participants failed to do the task and most of the participants performed it well $\left(d^{\prime}:\right.$ median $\left.=1.27\right)$. Participants used a rather conservative strategy, with a bias significantly different from 0 [Wilcoxon test, $Z(31)=488, p<0.0001 ; C$ : median $=0.55$; Figure 2C]. The median of minRTs across participants was $555 \mathrm{~ms}$ (range $=[390-780])$. Intriguingly, focusing on the fastest minRTs, 2 participants out of $31(6.5 \%)$ had a minRT at $390 \mathrm{~ms}$ (with the following bin at $420 \mathrm{~ms}$ being empty, Figure 2D). No correlation was found between $d^{\prime}$ values and minRTs $(\rho=-0.18$, $p=0.33$; Figure 2A). Across trials, median RT was $626 \mathrm{~ms}$ and minimal RT was $450 \mathrm{~ms}$. The RT distribution is presented in Figure 3.

\section{DISCUSSION}

This experiment is in agreement with a previous study (BarraganJason et al., 2012) and suggests that participants were rather slow overall since the median of minimal RTs was $555 \mathrm{~ms}$, despite the use of speed constraints. However, the variability of RTs across participants was high. Intriguingly, two participants appeared much faster than the rest of the group, yet showed comparable accuracy. They appeared even faster than the across-trial minRT. This suggests that the speed constraints we used in this experiment may not have been optimal. We therefore tried to constrain participants to use an even faster strategy in Experiment 2.

\section{EXPERIMENT 2: THE SAB PROCEDURE: EXTREME SPEED CONSTRAINTS}

A recent study introduced the Speed and Accuracy Boosting (SAB) procedure (Besson et al., 2012). This novel protocol is assumed to constrain participants to use their fastest strategy and could thus allow assessing whether speed constraints in Experiment 1 were optimal or not. Basically, the SAB relies on a response deadline and audio-feedbacks to constrain subjects to answer both quickly and accurately (Figure 1B). We also increased the number of subjects $(N=101)$ in order to determine a robust minimal RT.

\section{PARTICIPANTS}

A total of 101 participants (67 females) were included in this study (median age: 25, range: [18-30], all right-handers). All participants had normal or corrected-to-normal vision, volunteered, and gave their written informed consent to participate in the experiment.

\section{STIMULI}

Stimuli consisted of 140 grayscale photographs of famous faces (corresponding to the images that were recognized with the best accuracy in experiment 1) and 140 unknown faces chosen randomly in the set of 540 unknown faces that remained the same 
A
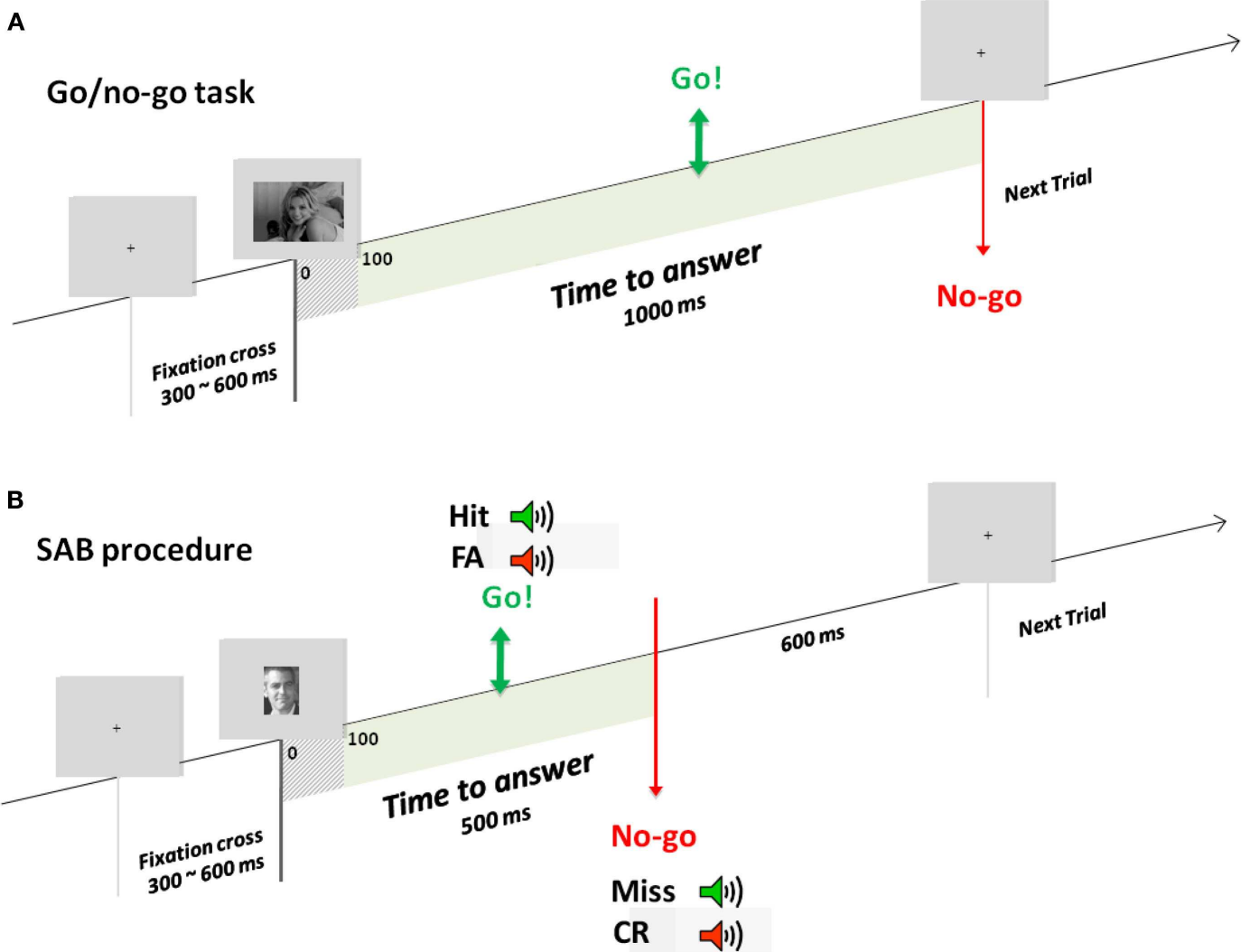

FIGURE 1 | Experimental designs. Example of one trial in the go/no-go task (A) used in experiment 1 and the SAB procedure $(\mathbf{B})$ used in experiment 2. During the go/no-go task, a fixation cross appeared for a random interval of 300-600 ms followed by the stimulus for $100 \mathrm{~ms}$ and a black screen which remained for $1000 \mathrm{~ms}$. In the SAB procedure, a fixation cross appeared for a random interval of 300-600 ms followed by the stimulus presentation. A gray screen remained for $600 \mathrm{~ms}$, which corresponded to the response deadline. If a go response was made before this response deadline, audio-feedback was played: positive if it was a target and negative if it was a distractor. In contrast, if a no-go response was made, positive audio-feedback was given at the response deadline if it was a distractor, negative if it was a target. for all participants. Because a previous study had shown that the SAB paradigm was highly demanding, all faces were cut out with the same size $\left(208 \times 279\right.$ pixels, visual angle: $\left.\sim 4.7 \times 6.3^{\circ}\right)$ from their context. Stimuli were presented one by one, in the center of a gray screen. Examples of stimuli are provided in Figure A1B in Appendix.

\section{THE SAB PROCEDURE}

Based on a classical go/no-go task, the SAB constrains participants to answer before a response deadline following stimulus onset (Figure 1B). Here, based on earlier results (Besson et al., 2012), we used a response deadline of $600 \mathrm{~ms}$ (almost no subject can perform the task with a deadline at $500 \mathrm{~ms}$ ). If a go response was made before this response deadline, an audio-feedback was played, positive if the item was a target (hit), negative if the item was a distractor (false-alarm). If a no-go response was made, audiofeedback was given at the response deadline, positive if the item was a distractor (correct no-go), or negative if the item was a target (omission). Before presentation of each item, a fixation cross was displayed for a pseudo-random time between 300 and $600 \mathrm{~ms}$. Items were presented for $100 \mathrm{~ms}$ (included in the response deadline). Each experiment was preceded by two training blocks (for each training block: 10 target stimuli, to be recognized among 10 distractor stimuli). The task was made of one block but a self-paced pause was proposed every 20 items. A pause of minimum $30 \mathrm{~s}$ was provided in the middle of the experiment (after 140 items).

\section{RESULTS}

The results across participants and across trials are presented in Table 1. Individual results are presented in Figures 2A,B. Some participants ( $N=14$, i.e., $13.9 \%$ ) failed to do the task, and their results were discarded. Participants used a slightly conservative strategy $[C$ : median $=0.07$; Wilcoxon test, $Z(101)=1403$, $p=0.02$; Figure 2C]. Median $d^{\prime}$ for the other 87 participants was 0.98. A minRT could be calculated for 57 of the 87 participants. The median of minRTs across participants was $420 \mathrm{~ms}$ (range $=[360$ 540]). A significant negative correlation was found between $d^{\prime}$ values and minimal RTs $(\rho=-0.52, p<0.0001$; Figure 2A). Across trials, median RT was $439 \mathrm{~ms}$ and minRT was $350 \mathrm{~ms}$. RT distribution is presented in Figure 3.

Compared to Experiment 1, minRTs were clearly shifted to the left (i.e., proportionally more participants performed at optimal speed, Figure 2D). Additionally, a significantly different 


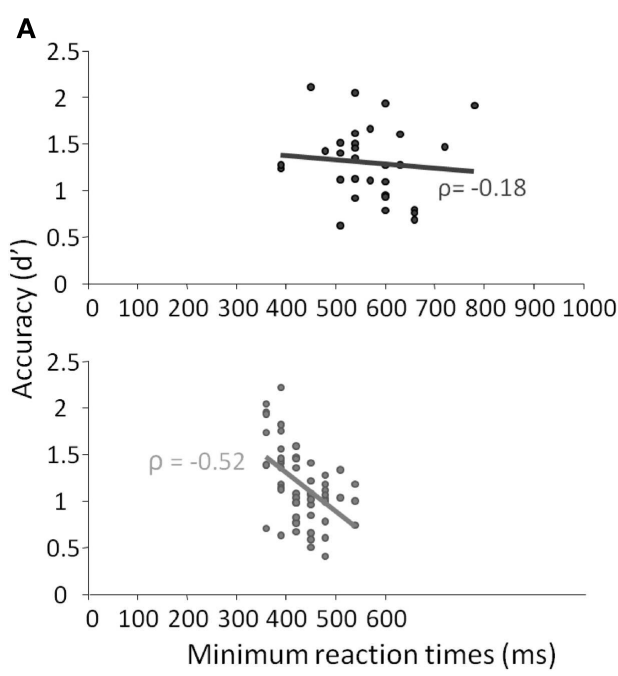

C

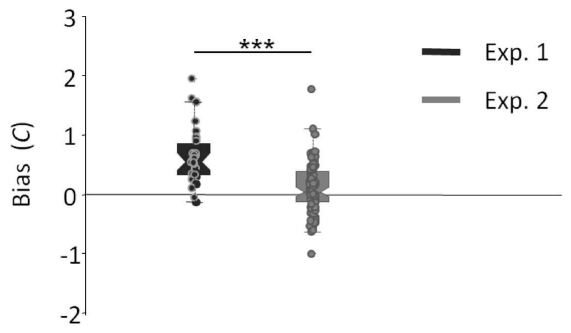

FIGURE 2 | Results from experiments 1 (dark gray) and 2 (light gray). (A) Minimal RTs for each participant according to $d^{\prime}$ and their correlation. Each point represents a participant. (B) $p$-Value of the Fisher exact test computed between the number of hits and the

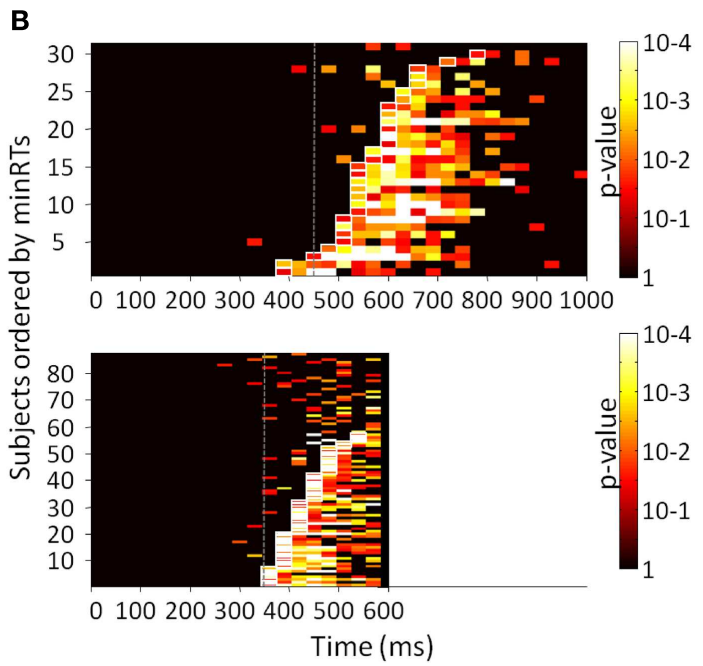

D

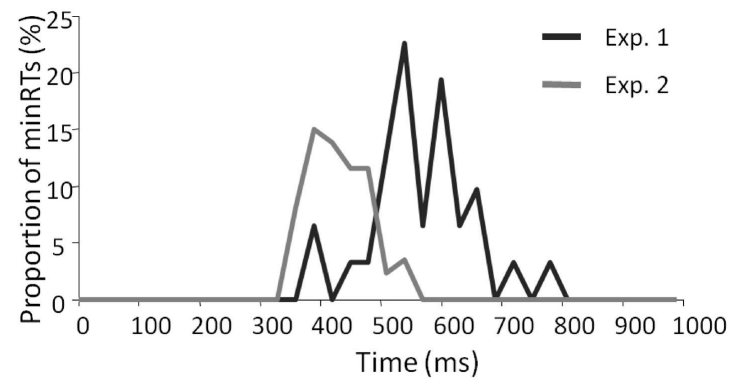

number of false alarms within each bin of $30 \mathrm{~ms}$. Lines represent individual participants, sorted by minRTs. (C) Boxplot of the bias (C) for both experiments. (D) Distribution of minimal RTs for both experiments. bias was observed between the two experiments, participants being more conservative in Experiment 1 than in Experiment 2 $[U(31)=2555, p<0.0001$; Figure 2C $]$.

\section{DISCUSSION}

The main aim of this experiment was to determine the minimum RT needed to recognize a face. With a large population, this experiment showed that (1) a large proportion of participants showed individual minRTs faster than $400 \mathrm{~ms}$; (2) $360 \mathrm{~ms}$ was the fastest individual minRT ( $350 \mathrm{~ms}$ across trials). Overall, this experiment brings strong evidence that face recognition is possible in $\sim 360 \mathrm{~ms}$ and probably not faster than this. However, and importantly, the fastest RTs were not much modified compared to Experiment 1 .

\section{GENERAL DISCUSSION}

The present study investigates the minimal time strictly necessary to recognize a face. A large number of famous faces were used in both experiments, preventing subjects from relying on arbitrary or idiosyncratic visual clues (i.e., specific hair styles) that would have facilitated recognition. Our experiments thus clearly assess the speed of bottom-up recognition. In Experiment 1, we showed that participants could spontaneously recognize famous faces in as little as $390 \mathrm{~ms}$ during a challenging go/no-go categorization task (range: [390-780]). A large variability in RTs was observed among participants however. Fast participants $(6.5 \%)$ could recognize faces in only $390 \mathrm{~ms}$, whereas slow participants needed roughly $165 \mathrm{~ms}$ more to perform the same task. In Experiment 2, we therefore employed the SAB procedure, which adds a response deadline (here at $600 \mathrm{~ms}$ post-stimulus onset) to speed up responses and an audio-feedback (i.e., positive or negative) to optimize accuracy in order to encourage participants to use their fastest strategy. This highly demanding task was efficient since participants recognized famous faces faster, with a smaller time range [360-540 ms], and without any speed/accuracy tradeoff. Across-trial minRTs shifted from 450 to $350 \mathrm{~ms}$. However, the gain in individual fastest minRTs was relatively small as it shifted by only $30 \mathrm{~ms}$, from 390 to $360 \mathrm{~ms}$, suggesting that the fastest participants in Experiment 1 were already (and spontaneously) close to optimum performance. Across the two experiments, and despite different conditions, it thus appears that human participants can recognize famous faces in as little as $360-390 \mathrm{~ms}$ and that they cannot perform faster. This is much longer than the time needed to detect faces in natural scenes (250-290 ms, reviewed in Fabre-Thorpe, 2011) using similar behavioral paradigms. This lower behavioral bound at $360 \mathrm{~ms}$ is also an upper bound for 


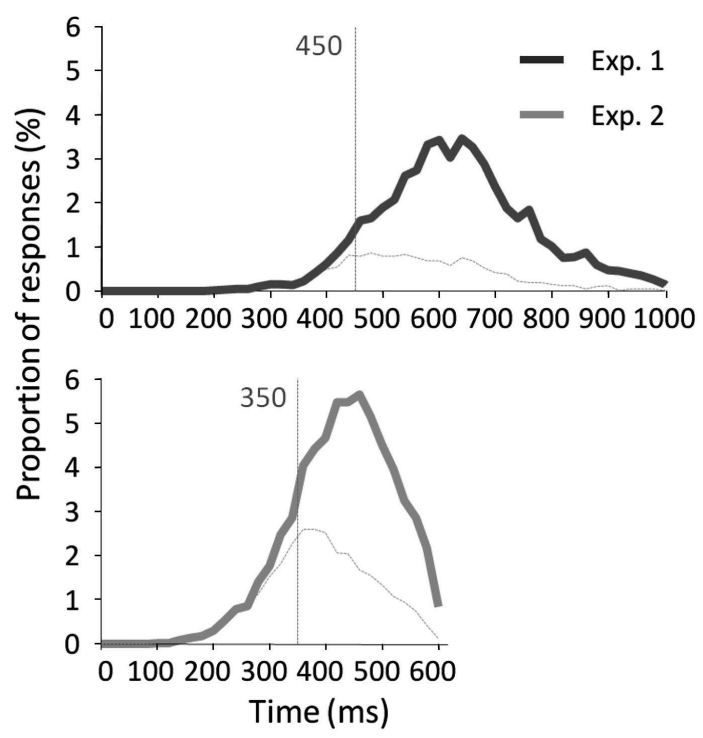

FIGURE 3 | RT distribution across trials with correct go-responses and false alarms for experiment $\mathbf{1}$ (top) and experiment $\mathbf{2}$ (bottom). Vertical lines indicate the minimal RT across trials.

neuronal processing. Given that about $100-130 \mathrm{~ms}$ are needed for decision and motor responses (Kalaska and Crammond, 1992; VanRullen and Thorpe, 2001b), this suggests that the fastest neural processes underlying face familiarity have occurred by $260 \mathrm{~ms}$. This is clearly not in the time-window of the well-known component peaking at $170 \mathrm{~ms}$ in $M / E E G$ studies and thought to index access to face representation. All in all, this suggests that face familiarity requires specific processes beyond face detection or access to face representation. These implications are discussed below.

Recognition memory has been largely described as being supported by two processes, familiarity and recollection (Mandler, 1980; Yonelinas and Levy, 2002; Yonelinas et al., 2010). The former (mere feeling that an item has been experienced previously) would be automatic and rapid, the latter (retrieval of specific contextual details) would be more effortful and time consuming (Juola et al., 1971; Brown and Aggleton, 2001; Rugg and Curran, 2007; Besson et al., 2012; Staresina et al., 2012). Therefore, we can hypothesize that a fast strategy relies merely on a feeling of familiarity, whereas a slow strategy involves the recollection of information about the person, which takes time. Most of the participants in Experiment 1 appeared to take some time to produce their responses compared to two "fast" participants. This appears to be in agreement with the idea that most are not satisfied by a mere feeling of familiarity but also need to identify the face (Bruce and Young, 1986; Valentine, 2001).

The SAB procedure was apparently successful at constraining participants to rely on a faster, familiarity-based, procedure since minRTs were clearly shifted toward a minimal boundary around 360-390 ms. Participants were found to be much less conservative in Experiment 2 than in Experiment 1 (Figure 2C), supporting the idea that participants used a different strategy. A conservative strategy would be expected if participants were allowed some time (even under speed constraints but without a response deadline) so that they could optimize the hit rate and diminish FA, which are socially inadequate. However, because stimuli were different between experiment 1 and 2, it is possible that just using stimuli of experiment 2 in experiment 1 would have generated the results observed with the SAB procedure. It could be interesting to verify this point in another study. Overall, both studies converge to a minimal boundary at 360-390 ms.

What can this 360-390 ms time limit tell us about underlying neural mechanisms? Considering the literature on rapid object categorization, it seems that about $250-290 \mathrm{~ms}$ are needed to produce reliable behavioral responses when categorizing visual objects (Fabre-Thorpe et al., 2001; VanRullen and Thorpe, 2001a; Rousselet et al., 2003; Joubert et al., 2007; Macé et al., 2009; FabreThorpe, 2011). Knowing that the time to trigger a manual response is around 100-130 ms (Kalaska and Crammond, 1992; VanRullen and Thorpe, 2001b), object categorization would rely on neural activity starting at about $120 \mathrm{~ms}$. Within this framework, Dicarlo et al. (2012) suggest that object recognition is consistent with feed-forward inter-area processing involving about 10 synapses from the retina to high level visual areas [infero-temporal cortex (IT)]. Considering that $10 \mathrm{~ms}$ are needed to transfer information between each of these different areas, Dicarlo et al. (2012) proposed that a first high level representation of the object can be available around $100 \mathrm{~ms}$ after its presentation (e.g., Desimone et al., 1984; Logothetis and Sheinberg, 1996; Liu et al., 2009). This feed-forward hypothesis is consistent with recent behavioral studies showing that participants can initiate saccades toward visual objects (faces, animals) in as little as $100-120 \mathrm{~ms}$ after stimulus onset (Kirchner and Thorpe, 2006; Crouzet et al., 2010).

Here, we find that recognizing faces takes $\sim 100 \mathrm{~ms}$ longer than object categorization. It could be proposed that face recognition relies on the same feed-forward mechanisms that have been posited for object categorization but with the recruitment of additional areas higher up in the visual stream. Visual recognition has traditionally been considered as a hierarchical process where visual representations become gradually more invariant and specific along the pathway. Familiarity with a face requiring the highest level of specificity, visual areas in the highest area of the visual ventral pathway, such as the perirhinal cortex or even the temporal pole, could be recruited for such a process. Evidence in favor of the implication of the anterior temporal lobes in person processing are numerous, including studies of brain-lesioned patients suffering from person agnosia (Joubert et al., 2004, 2006) and fMRI studies (e.g., Haxby et al., 2000). Furthermore, medial temporal lobe structures such as perirhinal and entorhinal cortex or hippocampus could be involved in these processes, as their role in recognition memory tasks has often been underlined (Bowles et al., 2007; Diana et al., 2007; Montaldi and Mayes, 2010; Staresina et al., 2012) and as it is also in these areas that "person-specific" neurons are found (Quiroga et al., 2005). The perirhinal cortex, in particular, is the highest area in the visual pathway and is thought to be a critical node for familiarity (Barbeau et al., 2005, 2011; Aggleton and Brown, 2006). 
If face recognition is only feed-forward, roughly 10 additional synapses should be activated. However, direct projections have been reported from IT to the perirhinal and temporal pole (Suzuki and Amaral, 1994a,b) so that the additional time necessary should be $20-30 \mathrm{~ms}$ at the very most. Thus, it appears implausible that face recognition should rely on a purely feed-forward process.

Another possibility, proposed by Hochstein and Ahissar (2002), distinguishes vision-at-a-glance based on feed-forward activity, from vision-with-scrutiny, based on processes beginning at the top of the hierarchy and "gradually returning as needed" to the ventral stream (Hochstein and Ahissar, 2002; Hegdé, 2008) in order to refine perceptual representations (Bullier et al., 2001). In line with this idea, an early activity in response to faces was observed at $130 \mathrm{~ms}$ in the anterior frontal gyrus, which could signal to areas at the top of the hierarchy that a face has been detected (Bar et al., 2006; Barbeau et al., 2008). Furthermore, a period of massively parallel processing has been identified in the whole visual ventral stream at $240 \mathrm{~ms}$ during a famous/unknown recognition task in intracerebral recordings in epileptic patients (Barbeau et al., 2008). Additionally, such latency could correspond to a differential activity between famous and unknown faces in the perirhinal cortex (Trautner et al., 2004). It is noteworthy that latencies in the hippocampus are delayed by about $80-100 \mathrm{~ms}$ compared to those of the perirhinal cortex (Trautner et al., 2004; Barbeau et al., 2008; Mormann et al., 2008). Considering the 100-130 ms necessary to trigger a manual response, this neural activity at $240 \mathrm{~ms}$ could be the basis for the first reliable behavioral responses at $360 \mathrm{~ms}$ observed in this study. Hence, face recognition would not rely on a first pass of feed-forward activity, but would more plausibly involve parallel processes implying both local and inter-area feed-back communication within and among the whole ventral stream.

An alternative can be formulated, however. A core network for face processing involving the Occipital Face Area (OFA), the Fusiform Face Area (FFA), and the posterior superior temporal sulcus has been identified and studied extensively (Haxby et al., 2000, 2001; Gobbini and Haxby, 2006). Because these areas, the FFA and OFA in particular, show an adaptation effect for identity, it has been proposed that this core network could be involved in representing individual faces (Rossion, 2008, 2009). Consequently, this core network could be sufficient to support face recognition. Furthermore, based on the study of patients with brain lesions, it has been proposed that this core network does not rely on feed-forward processing (from posterior occipital areas to the OFA to the FFA) but on connections from posterior areas to the FFA, then back to the OFA using reentrant loops (Rossion, 2008, 2009). Hence, the latency we observe for familiarity-based recognition could be due to the time needed for this reentrant processing to take place, for instance to refine visual information. We believe this is a reasonable alternative to the idea put forward above that face familiarity would require the whole visual ventral stream and, to our mind, this alternative merits further investigation. At this stage however, we note that the activity of this core network is probably reflected in the component peaking around $170 \mathrm{~ms}$ recorded using $M / E E G$. This component is supposed to reflect configural/holistic processes (e.g., Gosling and Eimer, 2011) but some studies suggest that it may also reflect individuation/recognition processes (e.g., Jemel et al., 2010). Behavioral latencies reported in the current study appear incompatible with this early component. Furthermore, such a scheme would discard the role of the perirhinal cortex in face recognition whereas, as already mentioned, this area appears to be a key node in familiarity, and faces elicit prominent activity in this region (Trautner et al., 2004; Dietl et al., 2005; Barbeau et al., 2008).

It should be noted that a potential limit to our study is that stimuli were different in Experiments 1 and 2, implying that performance of the two tasks cannot be directly compared. However, this is not a major problem since results in Experiments 1 and 2 are actually congruent on the idea that the fastest individual RTs occur around 360-390 ms, which is the main emphasis of this study. Although more subjects performed around 360 $390 \mathrm{~ms}$ in Experiment 2, the use of easier stimuli and stronger speed constraints did not allow them to be much faster than in Experiment 1. Additionally, previous studies that used different paradigms or type of stimuli reported behavioral latencies that seem consistent with our results (418 ms in Anaki et al., 2007; 380 ms in Ramon et al., 2011; 431 ms in Kampf et al., 2002). Anaki et al. (2007) and Kampf et al. (2002) studies were quite similar to our experiments: they used similar categories of faces (famous vs. unknown) and a comparable number of stimuli (180 targets vs. 180 distractors in Anaki et al. and 144 vs. 288 in Kampf et al.), i.e., subjects were in a bottom-up recognition situation. However, these authors used yes/no tasks rather than a go/no-go task as here; they didn't use any speed constraints and they focused on mean rather than minimal RTs. In contrast, Ramon et al. (2011) reported minimum RTs but a small set of faces was used (28 faces of classmates). The impact of this restricted set of faces on speed remains to be determined. These previous studies suggest that the lower bound for the fastest RTs were reached independently of the conditions and stimuli used, confirming our current results performed under speed constraints and in a bottom-up situation.

In conclusion, the current study has determined that the very minimal RT to recognize famous faces among unknown ones is around $360-390 \mathrm{~ms}$ after stimulus onset. It seems that such latencies are consistent with the speed of recognition memory in general (i.e., objects and abstract patterns, Besson et al., 2012), raising the question of whether the familiarity processes used to recognize faces are face-specific or are related to a general familiarity system. The SAB procedure seems an efficient method to determine the minimal time needed to perform a cognitive task. Such a method could be used to study other kinds of familiar faces, such as personally known faces (Ramon et al., 2011) or newly learned faces (Tanaka et al., 2006). We have discussed which model of activation of the visual ventral stream could account for such latencies. Three models were proposed and the one favored to date is that of an activation of the whole visual ventral stream up to anterior areas such as the perirhinal cortex, combined with parallel and feed-back processes. Further studies are needed to assess these three models of activation better. 


\section{REFERENCES}

Aggleton, J. P., and Brown, M. W. (2006). Interleaving brain systems for episodic and recognition memory. Trends Cogn. Sci. (Regul. Ed.) 10, 455-463.

Anaki, D., and Bentin, S. (2009). Familiarity effects on categorization levels of faces and objects. Cognition 111, 144-149.

Anaki, D., Zion-Golumbic, E., and Bentin, S. (2007). Electrophysiological neural mechanisms for detection, configural analysis and recognition of faces. Neuroimage 37, 1407-1416.

Bacon-Macé, N., Kirchner, H., FabreThorpe, M., and Thorpe, S. J. (2007). Effects of task requirements on rapid natural scene processing: from common sensory encoding to distinct decisional mechanisms. J. Exp. Psychol. Hum. Percept. Perform. 33, 1013-1026.

Baird, L. M., and Burton, A. M. (2008). The bilateral advantage for famous faces: interhemispheric communication or competition. Neuropsychologia 46, 1581-1587.

Bar, M., Kassam, K. S., Ghuman, A. S., Boshyan, J., Schmid, A. M., Schmidt, A. M., et al. (2006). Top-down facilitation of visual recognition. Proc. Natl. Acad. Sci. U.S.A. 103, 449-454.

Barbeau, E. J., Felician, O., Joubert, S., Sontheimer, A., Ceccaldi, M., and Poncet, M. (2005). Preserved visual recognition memory in an amnesic patient with hippocampal lesions. Hippocampus 15, 587-596.

Barbeau, E. J., Pariente, J., Felician, O., and Puel, M. (2011). Visual recognition memory: a double anatomofunctional dissociation. Hippocampus 21, 929-934.

Barbeau, E. J., Taylor, M. J., Regis, J., Marquis, P., Chauvel, P., and Liégeois-Chauvel, C. (2008). Spatio temporal dynamics of face recognition. Cereb. Cortex 18, 997-1009.

Barragan-Jason, G., Lachat, F., and Barbeau, E. J. (2012). How fast is famous face recognition? Front. Psychol. 3:454. doi:10.3389/fpsyg.2012.00454

Bentin, S., and Deouell, L. Y. (2000). Structural encoding and identification in face processing: ERP evidence for separate mechanisms. Cogn. Neuropsychol. 17, 35-55.

Besson, G., Ceccaldi, M., Didic, M., and Barbeau, E. J. (2012). The speed of visual recognition memory. Vis. Cogn. 20, 1131-1152.

Bowles, B., Crupi, C., Mirsattari, S. M., Pigott, S. E., Parrent, A. G., Pruessner, J. C., et al. (2007). Impaired familiarity with preserved recollection after anterior temporal-lobe resection that spares the hippocampus. Proc. Natl. Acad. Sci. U.S.A. 104, 16382-16387.

Brown, M. W., and Aggleton, J. P. (2001) Recognition memory: what are the roles of the perirhinal cortex and hippocampus? Nat. Rev. Neurosci. 2, 51-61.

Bruce, V., and Young, A. (1986). Understanding face recognition. Br. J. Psychol. 77, 305-327.

Bullier, J., Hupé, J. M., James, A. C., and Girard, P. (2001). The role of feedback connections in shaping the responses of visual cortical neurons. Prog. Brain Res. 134, 193-204.

Caharel, S., Bernard, C., Thibaut, F., Haouzir, S., Di Maggio-Clozel, C., Allio, G., et al. (2007). The effects of familiarity and emotional expression on face processing examined by ERPs in patients with schizophrenia. Schizophr. Res. 95, 186-196.

Caharel, S., Courtay, N., Bernard, C., Lalonde, R., and Rebaï, M. (2005). Familiarity and emotional expression influence an early stage of face processing: an electrophysiological study. Brain Cogn. 59, 96-100.

Crouzet, S. M., Kirchner, H., and Thorpe, S. J. (2010). Fast saccades toward faces: face detection in just 100 ms. J. Vis. 10, 1-17.

Desimone, R., Albright, T. D., Gross, C. G., and Bruce, C. (1984). Stimulusselective properties of inferior temporal neurons in the macaque. $J$. Neurosci. 4, 2051-2062.

Diana, R. A., Yonelinas, A. P., and Ranganath, C. (2007). Imaging recollection and familiarity in the medial temporal lobe: a three-component model. Trends Cogn. Sci. (Regul. Ed.) 11, 379-386.

Dicarlo, J. J., Zoccolan, D., and Rust, N. C. (2012). How does the brain solve visual object recognition? Neuron 73, 415-434.

Dietl, T., Trautner, P., Staedtgen, M., Vannuchi, M., Mecklinger, A., Grunwald, T., et al. (2005). Processing of famous faces and medial temporal lobe event-related potentials: a depth electrode study. Neuroimage 25, 401-407.

Fabre-Thorpe, M. (2011). The characteristics and limits of rapid visual categorization. Front. Psychol. 2:243. doi:10.3389/fpsyg.2011.00243

Fabre-Thorpe, M., Delorme, A., Marlot, C., and Thorpe, S. (2001). A limit to the speed of processing in ultrarapid visual categorization of novel natural scenes. J. Cogn. Neurosci. 13, 171-180.
Gobbini, M. I., and Haxby, J. V. (2006) Neural response to the visual familiarity of faces. Brain Res. Bull. 71, 76-82.

Gosling, A., and Eimer, M. (2011). An event-related brain potential study of explicit face recognition. $\mathrm{Neu}$ ropsychologia 49, 2736-2745.

Haxby, J. V., Gobbini, M. I., Furey, M. L., Ishai, A., Schouten, J. L., and Pietrini, P. (2001). Distributed and overlapping representations of faces and objects in ventral temporal cortex. Science 293, 2425-2430.

Haxby, J. V., Hoffman, E. A., and Gobbini, M. I. (2000). The distributed human neural system for face perception. Trends Cogn. Sci. (Regul. Ed.) 4, 223-233.

Hegdé, J. (2008). Time course of visual perception: coarse-to-fine processing and beyond. Prog. Neurobiol. 84, 405-439.

Herzmann, G., Schweinberger, S. R., Sommer, W., and Jentzsch, I. (2004). What's special about personally familiar faces? A multimodal approach. Psychophysiology 41, 688-701.

Hochstein, S., and Ahissar, M. (2002). View from the top: hierarchies and reverse hierarchies in the visual system. Neuron 36, 791-804.

Jemel, B., Schuller, A.-M., and Goffaux, V. (2010). Characterizing the spatio-temporal dynamics of the neural events occurring prior to and up to overt recognition of famous faces. J. Cogn. Neurosci. 22, 2289-2305.

Joubert, O. R., Rousselet, G. A., Fize, D., and Fabre-Thorpe, M. (2007). Processing scene context: fast categorization and object interference. Vision Res. 47, 3286-3297.

Joubert, S., Felician, O., Barbeau, E., Ranjeva, J.-P., Christophe, M., Didic, M., et al. (2006). The right temporal lobe variant of frontotemporal dementia: cognitive and neuroanatomical profile of three patients. J. Neurol. 253, 1447-1458.

Joubert, S., Felician, O., Barbeau, E., Sontheimer, A., Ceccaldi, M., and Poncet, M. (2004). A longitudinal case study of right temporal variant frontotemporal degeneration. Neurology 63, 1962-1965.

Juola, J. F., Fischler, I., Wood, C. T., and Atkinson, R. C. (1971). Recognition time for information stored in longterm memory. Percept. Psychophys 10, 8-14.

Kalaska, J., and Crammond, D. (1992). Cerebral cortical mechanisms of reaching movements. Science 255, 1517-1523.
Kampf, M., Nachson, I., and Babkoff, H. (2002). A serial test of the laterality of familiar face recognition. Brain Cogn. 50, 35-50.

Kirchner, H., and Thorpe, S. J. (2006). Ultra-rapid object detection with saccadic eye movements: visual processing speed revisited. Vision Res. 46, 1762-1776.

Lewis, M. B., and Ellis, H. D. (2000). The effects of massive repetition on speeded recognition of faces. Q. J. Exp. Psychol. A. 53, 1117-1142.

Liu, H., Agam, Y., Madsen, J. R., and Kreiman, G. (2009). Timing, timing, timing: fast decoding of object information from intracranial field potentials in human visual cortex. Neuron 62, 281-290.

Logothetis, N. K., and Sheinberg, D. L. (1996). Visual object recognition. Annu. Rev. Neurosci. 19, 577-621.

Macé, M. J.-M., Joubert, O. R., Nespoulous, J.-L., and FabreThorpe, M. (2009). The time-course of visual categorizations: you spot the animal faster than the bird. PLoS ONE 4:e5927. doi:10.1371/journal.pone.0005927

Mandler, G. (1980). Recognizing: the judgment of previous occurrence. Psychol. Rev. 87, 252-271.

Montaldi, D., and Mayes, A. R. (2010). The role of recollection and familiarity in the functional differentiation of the medial temporal lobes. Hippocampus 20, 1291-1314.

Mormann, F., Kornblith, S., Quiroga, R. Q., Kraskov, A., Cerf, M., Fried, I., et al. (2008). Latency and selectivity of single neurons indicate hierarchical processing in the human medial temporal lobe. J. Neurosci. 28, 8865-8872.

Quiroga, R. Q., Reddy, L., Kreiman, G., Koch, C., and Fried, I. (2005). Invariant visual representation by single neurons in the human brain. Nature 435, 1102-1107.

Ramon, M., Caharel, S., and Rossion, B. (2011). The speed of recognition of personally familiar faces. Perception 40, 437-449.

Rosch, E. (1975). Cognitive representations of semantic categories. J. Exp. Psychol. Gen. 104, 192.

Rossion, B. (2008). Constraining the cortical face network by neuroimaging studies of acquired prosopagnosia. Neuroimage 40, 423-426.

Rossion, B. (2009). "Clarifying the functional neuro-anatomy of face perception by single case neuroimaging studies of acquired prosopagnosia," in Cortical Mechanisms of Vision, eds L. Harris and M. Jenkin (Cambridge: Cambridge University Press), 171-207. 
Rousselet, G. A., Macé, M. J.-M., and Fabre-Thorpe, M. (2003). Is it an animal? Is it a human face? Fast processing in upright and inverted natural scenes. J. Vis. 3, 440-455.

Rugg, M. D., and Curran, T. (2007). Event-related potentials and recognition memory. Trends Cogn. Sci. (Regul. Ed.) 11, 251-257.

Snodgrass, J. G., and Corwin, J. (1988). Pragmatics of measuring recognition memory: applications to dementia and amnesia. J. Exp. Psychol. Gen. 117, 34-50.

Staresina, B. P., Fell, J., Do Lam, A. T. A., Axmacher, N., and Henson, R. N. (2012). Memory signals are temporally dissociated in and across human hippocampus and perirhinal cortex. Nat. Neurosci. 15, 1167-1173.

Suzuki, W. A., and Amaral, D. G. (1994a). Perirhinal and parahippocampal cortices of the macaque monkey: cortical afferents. J. Comp. Neurol. 350, 497-533.

Suzuki, W. A., and Amaral, D. G. (1994b). Topographic organization of the reciprocal connections between the monkey entorhinal cortex and the perirhinal and parahippocampal cortices. J. Neurosci. 14, 1856-1877.

Tanaka, J. W. (2001). The entry point of face recognition: evidence for face expertise. J. Exp. Psychol. Gen. 130, 534-543.

Tanaka, J. W., Curran, T., Porterfield, A. L., and Collins, D. (2006). Activation of preexisting and acquired face representations: the N250 event-related potential as an index of face familiarity. J. Cogn. Neurosci. 18, 1488-1497.

Thorpe, S., Fize, D., and Marlot, C. (1996). Speed of processing in the human visual system. Nature 381, 520-522.

Trautner, P., Dietl, T., Staedtgen, M., Mecklinger, A., Grunwald, T., Elger, C. E., et al. (2004). Recognition of famous faces in the medial temporal lobe: an invasive ERP study. Neurology 63, 1203-1208.

Valentine, T. (2001). "Face-space models of face recognition," in Computational, Geometric, and Process Perspectives on Facial Cognition: Contexts and Challenges, eds M. J. Wenger and J. T. Townsend (Mahwah: LEA), 83-113.

VanRullen, R., and Thorpe, S. J. (2001a). Is it a bird? Is it a plane? Ultra-rapid visual categorisation of natural and artifactual objects. Perception 30, 655-668.

VanRullen, R., and Thorpe, S. J. (2001b). The time course of visual processing: from early perception to decision-making. J. Cogn. Neurosci. 13, 454-461.

Yonelinas, A. P., Aly, M., Wang, W.C., and Koen, J. D. (2010). Recollection and familiarity: examining controversial assumptions and new directions. Hippocampus 20, 1178-1194.

Yonelinas, A. P., and Levy, B. J. (2002). Dissociating familiarity from recollection in human recognition memory: different rates of forgetting over short retention intervals. Psychon. Bull. Rev. 9, 575-582.

Yovel, G., and Paller, K. A. (2004). The neural basis of the butcheron-the-bus phenomenon: when a face seems familiar but is not remembered. Neuroimage 21, 789-800.

Zheng, X., Mondloch, C. J., and Segalowitz, S. J. (2012). The timing of individual face recognition in the brain. Neuropsychologia 50, 1451-1461.
Conflict of Interest Statement: The authors declare that the research was conducted in the absence of any commercial or financial relationships that could be construed as a potential conflict of interest.

Received: 23 November 2012; paper pending published: 28 December 2012; accepted: 11 February 2013; published online: 04 March 2013.

Citation: Barragan-Jason G, Besson $G$ Ceccaldi $M$ and Barbeau EJ (2013) Fast and famous: looking for the fastest speed at which a face can be recognized. Front. Psychol. 4:100. doi:10.3389/fpsyg.2013.00100

This article was submitted to Frontiers in Cognitive Science, a specialty of Frontier in Psychology.

Copyright (C) 2013 Barragan-Jason, Besson, Ceccaldi and Barbeau. This is an open-access article distributed under the terms of the Creative Commons Attribution License, which permits use, distribution and reproduction in other forums, provided the original authors and source are credited and subject to any copyright notices concerning any third-party graphics etc. 


\section{APPENDIX}

A
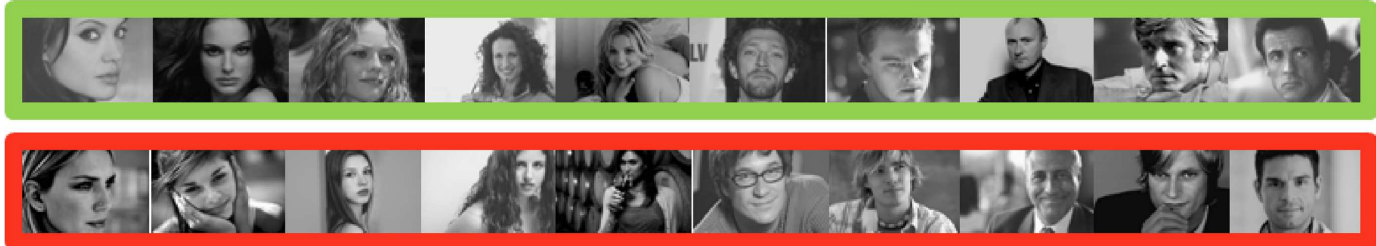

B
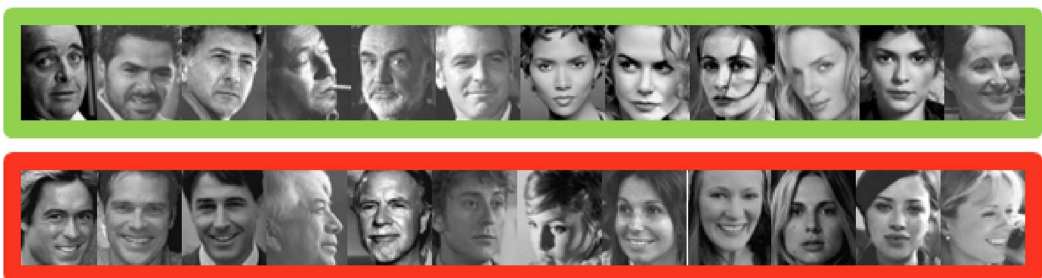

FIGURE A1 | Example of famous faces (green) and unknown faces (red) for experiment 1 in (A) and experiment 2 in (B). 